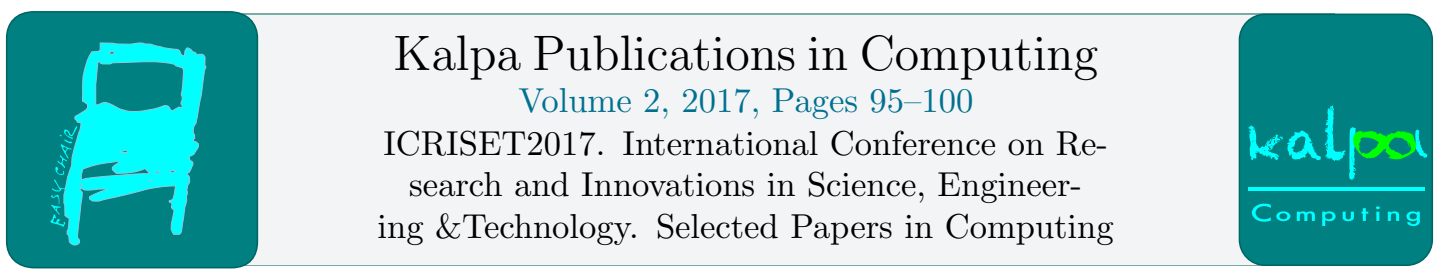

\title{
Soret and radiation effects on MHD chemically reactive Nano fluid flow over an exponentially accelerated vertical plate with ramped wall temperature and ramped surface concentration through porous medium.
}

\author{
Hari R. Kataria ${ }^{1}$ and Akhil S. Mittal ${ }^{2}$ \\ ${ }^{1}$ Department of mathematics, Faculty of science, The M. S. University of Baroda, Vadodara. \\ ${ }^{2}$ Department of mathematics, Gujarat Arts and science college, Ahmedabad. \\ hrkrmaths@yahoo.com, akhilsmittal@gmail.com
}

\begin{abstract}
Present paper is concerned with the study of flow, heat and mass transfer characteristics in the unsteady natural convective magneto hydrodynamics flow of chemically reactive electrically conducting Nano fluid past over an exponentially accelerated vertical plate in the presence of soret and thermal radiation. It is assumed that the bounding plate has ramped temperature with ramped surface concentration and isothermal temperature with ramped surface concentration profile through uniform porous medium. The governing non- dimensional partial differential equations are solved analytically using Laplace transform technique. The features of the fluid flow, heat and mass transfer are analyzed for all parameters by plotting graphs. Skin friction, Nusselt number and Sherwood number are obtained and represented through tabular form
\end{abstract}

\section{Introduction}

Nanofluids are colloidal suspensions of nanoparticles into a base liquid. So, a concentration flux is produced due to temperature gradient called Soret effect. Due to this effect, the concentration field will couple to temperature field described by the Soret coefficient. Sign of the Soret coefficient signifies movement of the nanoparticles to the colder lower boundary or to the warmer upper boundary. Mainly nanofluids are characterized by comparatively large negative Soret coefficient, hence the buoyancydriven motion is induced by the Soret diffusion even in initially uniformly stable thermal and concentration configuration. Makinde [1] has provided comprehensive discussion of MHD flow and 
heat transfer of Nano fluid. Heat and mass transfer effects on the magneto hydrodynamics of a flow of CNT based fluid over a convective surface was studied by Nadeem [2]. Kataria and Mittal [3-4] proposed Mathematical model for velocity and temperature of nanofluid flow past an oscillating vertical plate in presence of magnetic field and radiation. Effect of magnetic field on unsteady natural convective flow of a micropolar fluid between two vertical walls. Effect of magnetic field on unsteady natural convective flow of a micropolar fluid between two vertical walls is investigated by Kataria and Patel [5-6]. Kataria and Patel [7-8] discussed radiation and chemical reaction effects on MHD fluid flow past an oscillating vertical plate embedded in porous medium. To the best of author's knowledge, no attempt has been made to study the effect of the magnetic field, Soret and heat generation effects on MHD flow of radiating and chemically reactive Nano fluid past an oscillating vertical plate with ramped wall temperature and surface concentration through porous medium.

\section{Mathematical Formulation And Solution}

The flow is confined to $y>0$, where $y$ is the coordinate measured in the normal direction to the plate. The momentum and energy equations in the presence of thermal radiation and magnetic field past an oscillating vertical plate can be expressed as

$$
\begin{aligned}
& \rho_{n f} \frac{\partial u}{\partial t}=\mu_{n f} \frac{\partial^{2} u}{\partial y^{2}}-\sigma_{n f} B^{2} u-\frac{\mu_{n f} \varphi}{k_{1}} u+g(\rho \beta)_{n f}\left(T-T_{0}\right)+g\left(\rho \beta_{C}\right)_{n f}\left(C-C_{0}\right) \\
& \left(\rho c_{p}\right)_{n f} \frac{\partial T}{\partial t}=k_{n f} \frac{\partial^{2} T}{\partial y^{2}}-\frac{\partial q_{r}}{\partial y} \\
& \frac{\partial C}{\partial t}=D \frac{\partial^{2} C}{\partial y^{2}}+D_{T} \frac{\partial^{2} T}{\partial y^{2}}-k^{\prime}\left(C-C_{0}\right)
\end{aligned}
$$

Where

$$
\begin{aligned}
& \rho_{n f}=(1-\emptyset) \rho_{f}+\emptyset \rho_{s}, \mu_{n f}=\frac{\mu_{f}}{(1-\emptyset)^{2.5}}, \sigma=\frac{\sigma_{s}}{\sigma_{f}}, \quad \sigma_{n f}=\sigma_{f}\left[1+\frac{3(\sigma-1) \emptyset}{(\sigma+2)-(\sigma-1) \emptyset}\right], \\
& (\rho \beta)_{n f}=(1-\emptyset)(\rho \beta)_{f}+\emptyset(\rho \beta)_{s}, k_{n f}=k_{f}\left[1-3 \frac{\emptyset\left(k_{f}-k_{s}\right)}{2 k_{f}+k_{s}+\emptyset\left(k_{f}-k_{s}\right)}\right], \\
& \left(\rho c_{p}\right)_{n f}=(1-\emptyset)\left(\rho c_{p}\right)_{f}+\emptyset\left(\rho c_{p}\right)_{s}
\end{aligned}
$$

The initial and boundary conditions are

$$
\begin{aligned}
& u=\theta=C=0, \quad y \geq 0, t \leq 0 \\
& u=e^{a^{\prime} t}, \theta=\left\{\begin{array}{rr}
t, & 0<t \leq 1 \\
1 & t>1
\end{array}=t H(t)-(t-1) H(t-1),\right. \\
& C=\left\{\begin{array}{rr}
t, & 0<t \leq 1 \\
1 & t>1
\end{array}=t H(t)-(t-1) H(t-1) \text { at } y=0, t>0\right. \\
& u \rightarrow 0, \theta \rightarrow 0, C \rightarrow 0 \text { at } y \rightarrow \infty, t>0
\end{aligned}
$$

Introducing non dimensional variables

$$
\begin{aligned}
& y^{*}=\frac{u_{0} y}{v_{f}}, t^{*}=\frac{u_{0}^{2} t}{v_{f}}, u^{*}=\frac{u}{u_{0}}, \theta=\frac{\mathrm{T}-T_{0}}{T_{w}-T_{0}}, \omega^{*}=\frac{v_{f} \omega}{u_{0}^{2}}, C^{*}=\frac{C-C_{0}}{C_{w}-C_{0}} \\
& \frac{\partial u}{\partial t}=a_{1} \frac{\partial^{2} u}{\partial y^{2}}-\left(a_{3} M^{2}+\frac{a_{1}}{k}\right) u+a_{2} G_{r} \theta+G_{m} a_{5} C \\
& \frac{\partial \theta}{\partial t}=a_{4} \frac{\partial^{2} \theta}{\partial y^{2}} \\
& \frac{\partial C}{\partial t}=\frac{1}{s_{c}} \frac{\partial^{2} C}{\partial y^{2}}+S r \frac{\partial^{2} \theta}{\partial y^{2}}-k r C
\end{aligned}
$$




$$
\begin{aligned}
& C=\left\{\begin{array}{rr}
t, & 0<t \leq 1 \\
1 & t>1
\end{array}=t H(t)-(t-1) H(t-1) \text { at } y=0, t>0\right. \\
& u \rightarrow 0, \theta \rightarrow 0, C \rightarrow 0 \text { at } y \rightarrow \infty, t>0
\end{aligned}
$$

\subsection{Solution of the problem for ramped wall temperature and ramped surface concentration:}

$$
\begin{aligned}
& \theta(y, t)=f_{8}(y, t)-f_{8}(y, t-1) H(t-1) \\
& C(y, t)=h_{4}(y, t)-h_{4}(y, t-1) H(t-1) \\
& u(y, t)=f_{1}(y, t)+h_{1}(y, t)-h_{1}(y, t-1) H(t-1)
\end{aligned}
$$

\subsection{Solution of the problem for isothermal temperature and ramped surface concentration}

In order to understand effects of ramped temperature of the plate on the fluid flow, we must compare our results with isothermal temperature. In this case, the initial and boundary conditions are the same excluding Eq. (10) that becomes $\theta=1$ at $y=0, t \geq 0$. We find the isothermal temperature $\theta(y, t)$ using Laplace transform.

$$
\begin{aligned}
& \theta(y, t)=f_{7}(y, t) \\
& C(y, t)=f_{12}(y, t)-f_{12}(y, t-1) H(t-1)+g_{12}(y, t) \\
& u(y, t)=f_{1}(y, t)+h_{2}(y, t)+h_{3}(y, t)-h_{3}(y, t-1) H(t-1)
\end{aligned}
$$

\subsection{Nusselt Number, Sherwood Number and Skin friction:}

Expressions of Nusselt number $\mathrm{Nu}$, Sherwood Number Sh and Skin friction $\tau$ for both all cases are calculated from equations (11) to (16) respectively using the relation

$$
N_{u}=-\left(\frac{\partial \theta}{\partial y}\right)_{y=0}, s_{h}=-\left(\frac{\partial C}{\partial y}\right)_{y=0} \text { and } \tau^{*}(y, t)=-\left.\frac{\partial u}{\partial y}\right|_{y=0},
$$

For ramped wall temperature and ramped surface concentration:

$$
\begin{aligned}
& N u=-\left[I_{8}(t)-I_{8}(t-1) H(t-1)\right] \\
& S h=-\left[I_{29}(t)-J_{29}(t-1) H(t-1)\right] \\
& \tau=I_{1}(t)+I_{26}(t)-I_{26}(t-1) H(t-1)
\end{aligned}
$$

For isothermal temperature and constant surface concentration:

$$
\begin{aligned}
& N u=-\left[I_{7}(t)\right] \\
& S h=-\left[I_{12}(t)-I_{12}(t-1) H(t-1)+I_{25}(t)\right] \\
& \tau=I_{1}(t)+I_{27}(t)+I_{28}(t)-I_{28}(t-1) H(t-1)
\end{aligned}
$$

\section{Result Discussion And Conclusions}

The expressions for the velocity, temperature and concentration have been obtained in closed form with the help of the Laplace transform technique. The effects of the pertinent parameters on velocity, 
concentration and temperature profiles are presented graphically through figures 2 to 4 and Table 1 . The influences of the same parameters on the skin friction and rate of heat transfer at the plate are also discussed in details. The most important concluding remarks can be summarized as follows:

- Nanofluid velocity is getting accelerated with the progress of Grashof number Gr, mass Grashof number $\mathrm{Gm}$, phase angle $\omega t$ and time $\mathrm{t}$ while decreases with increase in Prandtl number $\mathrm{Pr}$, magnetic parameter $\mathrm{M}$ and Schimdt number Sc. Nanofluid velocity decreases with increase in volume fraction parameter $\emptyset$. Nanofluid velocity is more for isothermal temperature compared to ramped plate.

- Nanofluid temperature tends to increase with rise in values of Prandtl number Pr and time t.

- Concentration increases with increase in Schimdt number Sc and progress of time.

- Nusselt number $\mathrm{Nu}$ decreases on increasing $\mathrm{t}$ for ramped temperature and otherwise for isothermal case. Sherwood number Sh decreases with increase in t or Schimdt number Sc for both ramped temperature and isothermal plate. Skin friction and Nusselt number $\mathrm{Nu}$ increases with increase in volume fraction parameter $\emptyset$.

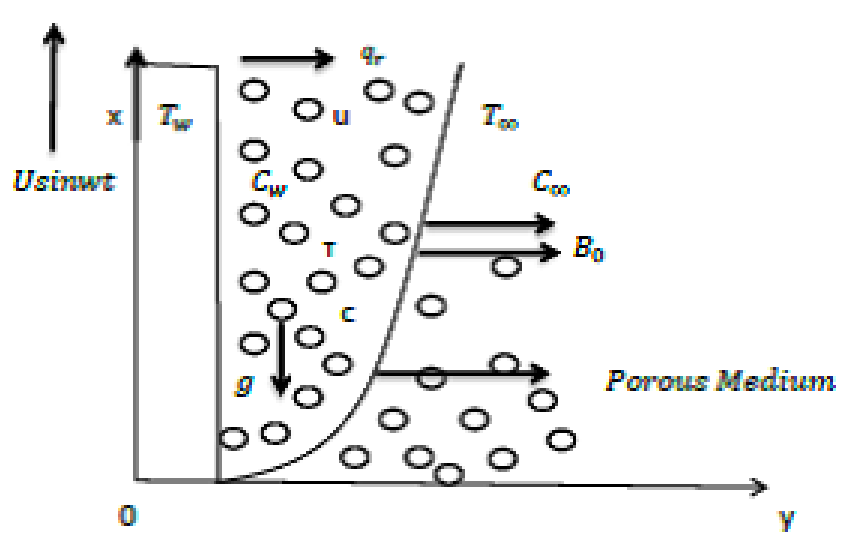

Figure 1: Physical sketch of the problem 


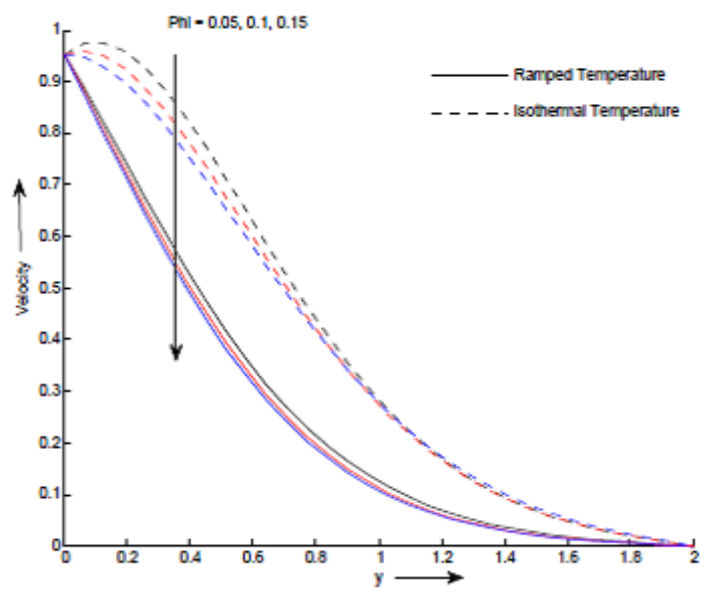

Figure 2: Velocity profile for different values of y \& Phi

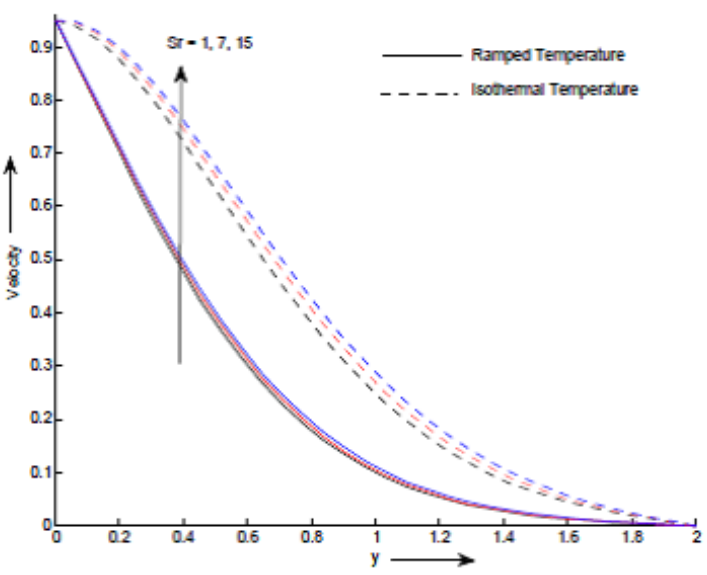

Figure 3: Velocity profile for different values of y $\& \mathrm{Sr}$

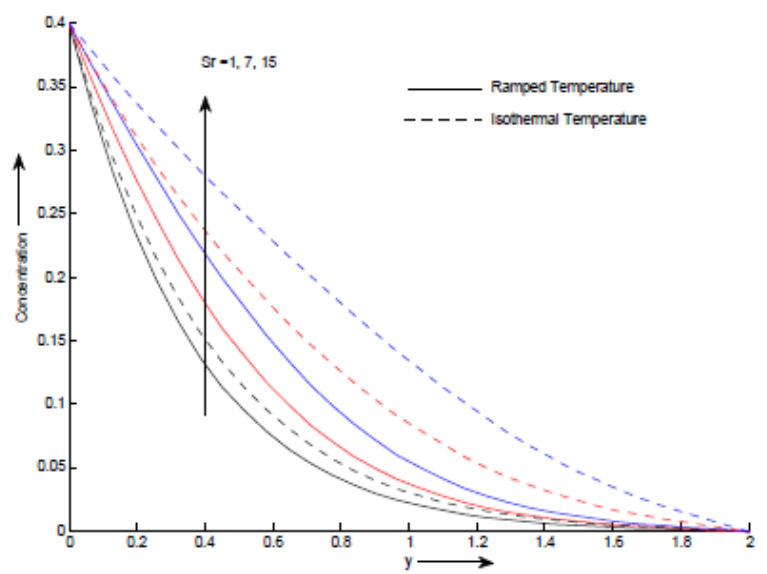

Figure 4: Concentration profile for different values of y \& Sr 
Table 1: Skin Friction, Nusselt Number And Sherwood Number

\begin{tabular}{|c|c|c|c|c|c|c|c|c|c|}
\hline$\$$ & $\bar{k}$ & $\mathrm{Sc}$ & $\mathrm{Sr}$ & $\begin{array}{l}\tau \text { For } \\
\text { Ramp. } \\
\text { Temp. }\end{array}$ & $\begin{array}{l}\tau \text { For } \\
\text { Const. } \\
\text { Tenp. }\end{array}$ & $\begin{array}{l}\text { Nu For } \\
\text { Ranp. } \\
\text { Temp. }\end{array}$ & $\begin{array}{l}\text { Nu For } \\
\text { Const. } \\
\text { Temp. }\end{array}$ & $\begin{array}{l}\text { Sh For } \\
\text { Ramp. } \\
\text { Temp. }\end{array}$ & $\begin{array}{l}\text { Sh For } \\
\text { const. } \\
\text { Temp. }\end{array}$ \\
\hline 0.05 & 1.0 & 1.2 & 3 & -3.9942 & 0.8039 & 0.0790 & -1.6599 & -3.9942 & 0.4883 \\
\hline 0.06 & 1.0 & 1.2 & 3 & -4.6395 & 0.4372 & 0.0767 & -1.6105 & -4.6395 & 0.4796 \\
\hline 0.07 & 1.0 & 1.2 & 3 & -5.5781 & -0.1387 & 0.0744 & -1.5629 & -5.5781 & 0.4663 \\
\hline 0.05 & 1.1 & 1.2 & 3 & -4.0526 & 0.9129 & 0.0790 & -1.6599 & -3.9942 & 0.4883 \\
\hline 0.05 & 1.2 & 1.2 & 3 & -4.1553 & 09913 & 0.0790 & -1.6599 & -39942 & 0.4883 \\
\hline 0.05 & 1.1 & 1.3 & 3 & -3.8424 & 0.9935 & 0.0790 & -1.6599 & -3.8424 & 0.4728 \\
\hline 0.05 & 1.1 & 1.4 & 3 & -3.7157 & 1.1497 & 0.0790 & -1.6599 & -3.7157 & 0.4562 \\
\hline 0.05 & 1.1 & 1.2 & 3.1 & -4.1226 & 0.8325 & 0.0790 & -1.6599 & -4.1226 & 0.5032 \\
\hline 0.05 & 1.1 & 1.2 & 3.2 & -4.2511 & 0.8612 & 0.0790 & -1.6599 & -4.2511 & 0.5181 \\
\hline
\end{tabular}

\section{References}

[1] O. D. Makinde, W. A. Khan, Z. H. Khan: Buoyancy effects on MHD stagnation point flow and heat transfer of a nanofluid past a convectively heated stretching/shrinking sheet. International Journal of Heat and Mass Transfer 62 (2013) 526-533.

[2] S Nadeem, R Mehmood, NS Akbar Oblique stagnation point flow of CNT based fluid over a convective surface Journal of Computational and Theoretical Nanoscience 12, 1-8, 2015.

[3] H. R. Kataria, A. S. Mittal, Velocity, mass and temperature analysis of gravity-driven convection nanofluid flow past an oscillating vertical plate in presence of magnetic field in a porous medium, Applied Thermal Engineering, 110 (2017) 864-874.

[4] H. R. Kataria, A. S. Mittal, Mathematical model for velocity and temperature of gravitydriven convective optically thick nanofluid flow past an oscillating vertical plate in presence of magnetic field and radiation. Journal of Nigerian Mathematical Society, 34 (2015) 303-317.

[5] H. R. Kataria, H. R. Patel, R. K. Singh, Effect of magnetic field on unsteady natural convective flow of a micropolar fluid between two vertical walls. Ain Shams Engineering Journal, DOI:10.1016/j.asej.2015.08.013.

[6] H. R. Kataria, H. R. Patel, Effect of thermo-diffusion and parabolic motion on MHD Second grade fluid flow with ramped wall temperature and ramped surface concentration, Alexandria Engineering Journal, 10.1016/j.aej.2016.11.014.

[7] H. R. Kataria, H. R. Patel, Radiation and chemical reaction effects on MHD Casson fluid flow past an oscillating vertical plate embedded in porous medium, Alexandria Engineering Journal , 55 (2016) 583-595.

[8] H. R. Kataria, H. R. Patel, soret and heat generation effects on MHD casson fluid flow past an oscillating vertical plate embedded through porous medium, Alexandria Engineering Journal, 55 (2016) 2125-2137. 